\title{
Endometriosis perineal: Otra causa de dolor perineal crónico
}

\author{
Edelberto Molett Vásqoez MD.*; María del Pilar Arango Arango MD.**
}

Recibido: Agosto 1 I 2000

Revisado: Agosto 4 I 2000

Aceptado: Agosto 11 I 2000

\section{RESUMEN}

La endometriosis perineal es una causa poco frecuente de consulta, pero se constituye en una importante causa de dolor perineal; una vez se tiene en cuenta en el diagnóstico diferencial, no es difícil hacer el abordaje diagnóstico e igualmente el tratamiento.

PALABRAS CLAVES: Endometriosis. Periné. Esfínter anal.

\section{SUMMARY}

Perineal endometriosis is an infrequent cause of medical consult, but is an important cause of chronic perineal pain. If the diagnosis is advised is not difficult the diagnostic approach and the treatment.

KEY IVORDS: Endometriosis. Perineal zone. Anal sphincter.

\section{Introducción}

La endometriosis es una de las entidades ginecológicas más frecuentes en la mujer; para la población general, se tiene una prevalencia de 2-4\% Y en pacientes con infertilidad y dolor pélvico puede ascender hasta un 10-25\% (1).

En algunas ocasiones la endometriosis también puede manifestarse con sintomatología extragenital, principalmente como dolor rectal y pélvico, de diferentes grados de severidad, sangrado rectal cíclico y trastornos intestinales asociados con la menstruación (2); mas raramente se puede presentar con síntomas obstructivos (3).

\section{Sintomatología}

Con relación a la endometriosis perineal, esta se puede considerar en términos generales como parte de la endometriosis que aparece en las cicatrices quirúrgicas, teniendo como etiología la implantación directa de células endometriales en la herida quirúrgica, después del parto vaginal y especialmente después de la exploración uterina manual o en el legrado postparto (4). En algunos casos, se puede también originar en el transporte de células endometriales por vía ven osa o linfática hasta el periné, sin que exista antecedente de trauma perineal previo (5).

Los síntomas de la endometriosis son causados por el crecimiento del tejido endometrial, causado por el estímulo hormonal, seguido por el sangrado al final del ciclo (4). Específicamente en la región perineal o perianal, la enfermedad se puede manifestar como dolor cíclico, masa dolorosa o sangrado intermitente en un área de cicatriz quirúrgica o traumática, edema o prurito (6-8).

\section{Diagnóstico}

La historia clínica puede orientar hacia el diagnóstico pero se han utilizado diferentes métodos para complementar el estudio clínico; estos principalmente son:

Ultrasonografía anal, tomografía axial computadorizada, resonancia magnética y la punción-aspirado de la lesión (9).

La ultrasonografía endoanal está indicada en la evaluación de las patologías benignas o malignas del recto y región anal o perianal, permitiendo la evaluación de la musculatura esfinteriana y los tejidos perianales (10).

La tomografía axial computada y la resonancia magnética nuclear, se han utilizado también como métodos diagnósticos adicionales para el estudio de las lesiones de los tejidos blandos perineales.

La punción-biopsia, se ha utilizado también para obtener el diagnóstico histológico de las lesiones sospechosas(11).

\section{Tratamiento}

Aunque en algunos casos se ha intentado el tratamiento supresivo hormonal, el tratamiento de elección es la resección quirúrgica y la reparación del esfínter o esfinteroplastia, en caso de haber compromiso del aparato esfinteriano (12-13). La reparación del esfínter se hace con la técnica de sobreposición (overlapping sphincteroplasty) de los extremos que quedan del esfínter externo seccionado, después de la excisión del endometrioma (14).

\footnotetext{
* Cirujano General. Cirujano de Colon y Recto. Profesor de Cirugía. Departamento Clínico - Quirúrgico. Facultad de Ciencias para la salud.

** Residente III año Ginecología y Obstetricia. Departamento Materno - Infantil. Facultad de Ciencias para la Salud. Universidad de Caldas
} 


\section{BIBLIOGRAFIA}

1. Botero W. Diagnóstico. En: Agudelo B. Aristizábal l, Botero W, et al. Simposio sobre endometriosis. Departamento de Ginecología y Obstetricia, Facultad de Medicina, Universidad de Antioquia. pp: 48-56.

2. Bailey HR. Colorectal endometriosis. Perspectives in Colon \& Rectal Surgery 1992; 5: 251-258.

3. Gordon PH. Miscellaneuus Entities. In Gordon PH, Nivatvongs S (cds). Principies and Practice of Surgery for the Colon Rectum, and Anus. QMP, 1992; 1041-1067.

4. Bacher H. Schweiger W, Cerwenka H, et al. Use of Anal Endosonography in Diagnosis of Endometriosis of the External Anal Sphincter. Report of a Case. Dis Colon Rectum 1999: 42: 680- 682.

5. Pollack R, Gordon PH, Ferenczy A, et al. Perineal endometriosis. A case reporto 1 Reprod Med 1990; 35: 109-12.

6. Hambrick E, Abcarian H, Smith D. Perineal endometrioma in episiotomy incisions: clinical features and management. Dis Colon Rectum 1979; 22: 550-2.

7. Salamalekis E, Vasiliadis TX, Kairi p, et al. Perineal endometriosis. Int J Gynaecol Obstet 1990; 31: 75-80.
8. Koger KE, Shatney CH, Hodge al. Surgical Scar Endometrioma. Surg Gynecol Obstet 1993; 177: 243-246.

9. Liang CC, Tsai CC. Chen TC, et al. Management of perineal endometriosis. Int J Gynaecol Obstet 1996; 53: 261-5.

10. Nogueras JJ. Endorectal Ultrasonography:

Technique Image Interpretarían, and Expanding Indications in 1995. Seminars in Colon \& Rectal Surgery 1995; 6: 70-77.

11. Puerta JD, Castaño R, Hoyos S. Endometrioma del Esfínter Anal. Rev Colomh Cir 1999; 14: 243-246.

12. Com1an ML. Less Common Tumors and Tumorlike Lesions of the Colon, Rectum, and Anus. In Colon \& Rectal Surgery. Third Edition. J.B. Lippincot Company. Philadelphia. 1993; 741-816.

13. Sayfan J, Benosh L, Segal M, et al. Endometriosis in episiotomy scar with anal sphincter involvement. Report of a case. Oís Colon Rectum 1991; 34: 713-6.

14. Tement CA. Direct Sphincter and Pelvic floor Reconstruction. Seminars in Colon \& Rectal Surgery 1997; 8: 93-102. 